Published in final edited form as:

Neurol Sci. 2008 September ; 29(4): 279-283. doi:10.1007/s10072-008-0982-6.

\title{
Varicella Zoster Virus Meningo-Encephalo-Myelitis in an Immunocompetent Patient
}

\author{
Eleonora Tavazzi, MD, \\ Department of Clinical Neurology, "C. Mondino" IRCCS Foundation, Pavia, Italy \\ Lorenzo Minoli, Professor, \\ Department of Infectious Diseases, "Policlinico San Matteo" IRCCS Foundation, Pavia, Italy \\ Pasquale Ferrante, Professor, \\ Laboratory of Virology, Department of Biomolecular Science and Technology, University of Milan, \\ Milan, Italy \\ San Giuseppe Hospital, Milan, Italy \\ Paola Scagnelli, MD, \\ Department of Neuroradiology, "Policlinico San Matteo" IRCCS Foundation, Pavia, Italy \\ Serena Del Bue, PhD, \\ Laboratory of Virology, Department of Biomolecular Science and Technology, University of Milan, \\ Milan, Italy
}

Alfredo Romani, MD, Department of Clinical Neurology, "C. Mondino" IRCCS Foundation, Pavia, Italy

Sabrina Ravaglia, MD,PhD, and Department of Clinical Neurology, "C. Mondino" IRCCS Foundation, Pavia, Italy

Enrico Marchioni

Department of Clinical Neurology, "C. Mondino" IRCCS Foundation, Pavia, Italy

\section{Abstract}

Objective-To describe clinical, MRI and cerebrospinal fluid (CSF) features of a varicella zoster virus (VZV) related meningo-encephalo-myelitis (MEM) without rash in an immunocompetent female.

Patient-An 85 year old immunocompetent woman with mild hyperthermia and acute, severe MEM.

Intervention-Serum antibodies and CSF PCR were searched for several viruses. Brain and spinal cord MRI were performed. Immunological profile.

Treatments-i.v. acyclovir $30 \mathrm{mg} / \mathrm{kg} /$ day; i.v. 6-MP $125 \mathrm{mg} /$ day.

Results-Marked CSF lymphomonocytic pleocytosis, blood-brain-barrier damage, and PCR detection of 3.05 X 106 copies of VZV DNA. MRI revealed lesions of the meninges, brain and spinal cord. No evidence of immunosuppression.

Corresponding author: Enrico Marchioni, MD, IRCCS Neurological Institute, via Mondino, 2, Pavia, It 27100 Italy, Tel: (+39-0382) 380225, Fax: (+39-0382) 380206, Email: enrico.marchioni@mondino.it.

Financial Disclosure: The authors report no conflicts of interest 
Conclusion-We highlight the importance of considering the possibility of VZV related MEM, even in immunocompetent patients. We also provide a MRI description of VZV related multifocal myelitis, not previously available. As supported by other reports, we underline the necessity of a prompt therapeutic intervention in this life-threatening condition

\section{Keywords}

varicella-zoster; meningitis; encephalomyelitis; immunocompetence; vasculitis

\section{Introduction}

Varicella-zoster virus (VZV) is known to be responsible for a broad spectrum of neurological diseases, ranging from the most common complication of zoster, namely post-herpetic neuralgia, to forms of neuritis, encephalitis, myelitis, ventriculitis and meningitis $(1,2)$. The immune setting of the patient affects the target, the spreading pathways and the clinical presentation of VZV-related CNS complications. Immunocompetent patients usually present with rash, possibly followed by satellite focal large vessel vasculitis. In contrast, immunocompromised subjects may show diffuse multifocal small vessel vasculitis due to hematogenous spread, and usually do not exhibit cutaneous manifestations (3). Simultaneous involvement of brain, spine and meninges in response to VZV reactivation is rare in general, and exceptional on a background of immunocompetence $(2,4)$.

Since 1997, we have been conducting a prospective study of infectious and post-infectious encephalomyelitis, with an extensive PCR analysis for viral genomes in CSF (5). One of the 92 immunocompetent patients showed MRI and laboratory findings consistent with a form of small-vessel vasculitis, fulfilling criteria for VZV MEM, and is the subject of this report.

\section{Case Report}

An 85-year-old, previously healthy woman presented with a subacute onset of headache, mild hyperthermia, confusion and seizures. She was admitted to an outside hospital where she underwent a brain CT, without relevant findings. The neurological examination showed altered consciousness, aphasia, monolateral facial palsy and a severe paraparesis of the lower limbs. Routine hematochemical tests were normal except for moderate leukocytosis $(22.000 / \mathrm{mm} 3)$ with relative neutrophilia (74\%). Limpid, high pressure CSF showed lymphocytic pleocytosis (700 cells/mmc, n.v. <2), elevated proteins $(990 \mathrm{mg} / \mathrm{dl})$ and severe blood brain barrier breakdown. Valproate was started (300 mg twice a day). An HIV screen was negative, and a neoplastic marker panel (CEA, Ca 19.9, Ca 125, Ca 15.3, alfa-fetoprotein, beta2microglobulin) and lymphocytic profile were both within normal limits. No other causes of immunological impairment were found. Chest X-ray was normal. One week after the onset of the symptoms, she was admitted to the Department of Infectious Diseases of Fondazione IRCCS Policlinico "San Matteo"; she presented comatose, with neck stiffness, myoclonic jerks and flaccid tetraplegia. Brain and spine MRI (see figs. 1a, b, c) showed multiple small T2 weighted hyperintense lesions and a marked and diffuse thickening of the meninges was observed with contrast enhancement at every level of the CNS. The patient also underwent another spinal tap in which CSF was hemorrhagic, showing marked pleocytosis (300 cells/ mmc: $92.8 \%$ lymphomonocytoid elements, $6.8 \%$ granulocytic elements) and hyperproteinorrachia (953 mg/dl). Real Time PCR analysis (6) was performed on CSF, searching for an extensive panel of viruses (CMV, EBV, JCV, VZV, HSV 1,2,6) (Marchioni et al, Neurol. 2005). Here, $3.05 \times 10^{6}$ copies/ml of VZV-DNA were found. Intravenous (i.v.) acyclovir $10 \mathrm{mg} / \mathrm{kg} /$ day divided q8h and i.v. steroid (6-methylprednisolone $125 \mathrm{mg} /$ day) were started. Two weeks later the patient died for systemic complications, after a partial neurological improvement. 


\section{Comments}

This is, to our knowledge, the first case in the literature describing a probable VZV small vessel vasculitis in an immunocompetent patient with MRI evidence of concurrent involvement of meninges, brain and spine. Unfortunately we do not have autopsy confirmation, but a form of small-vessel vasculopathy could be reasonably hypothesized, given the clinical presentation and the strongly suggestive MRI (i.e. subacute onset and multifocal lesions located in typical sites). Differential diagnosis should take into account other causes of vasculitis. Primary angiitis of the CNS and systemic arteritis could present a similar clinical and MRI patterns (7), but the detection of a marked CSF VZV viral load and the lack of specific auto-antibodies make this hypothesis improbable. Post infectious Acute Disseminated Encephalomyelitis (ADEM) can follow systemic VZV infections, but only occasionally can be induced by direct CNS VZV infection. It is characterized by a different clinical, CSF and MRI pattern. ADEM generally occurs after a florid infection, with CSF abnormalities consisting of mild to moderate pleocytosis and blood barrier damage and, finally, brain and spine MRI lesions, located in the deep white matter and in the basal ganglia. Moreover, in ADEM, meninges and cortex are usually spared. This is contrary to what we observed in our case. According to available data VZV small vessel vasculitis was not preceded by zoster. Convincing findings (HIV not detectable, no evidence of solid or hematological neoplasms, normal immunological profile), led us to consider this patient as immunocompetent, even though this form of infection is generally considered to exclusively affect immunocompromised patients. This atypical setting could justify the delay in performing appropriate investigations and in starting the antiviral treatment, thereby reducing the possibility of survival. The pathogenetic mechanism could be the reactivation of the virus within the trigeminal ganglion, followed by meningeal dissemination, which could explain violent headache as the initial symptom. A subsequent hematogeneous invasion could lead to diffuse, multifocal brain and spinal cord involvement, probably facilitated by a physiological, age-related lack of immune defences. Being elderly by itself is usually considered a risk factor for zoster, but no data are known about the role of this factor in small vessel VZV related complications (8). The majority of VZV vasculopathies is sustained by large vessel vasculitis, characterized by acute onset of focal deficits with congruent brain or spine MRI findings of focal ischemia. Instead, small vessel vasculopathy involves intraparenchymal arterioles producing multifocal lesions at the grey-white matter junction and in the deep white matter (2). Histopathologically, this vasculopathy is characterized by various degrees of CNS damage, ranging from demyelination to necrosis (9). In the literature there are few reports of VZV encephalo-myelitis (table); only two of them were in immunocompetent patients $(10,11)$, with one undergoing brain MRI, while the other had only a brain CT, both of which demonstrated normal results. According to our observation we can affirm that the MRI pattern of VZV small vessel myelitis is characterized by multifocal, monometameric, disseminated lesions with meningeal enhancement. However, to date, analogous MRI reports are lacking in the literature, so we can assume as a reference only pathological data (2). Kleinschmidt-DeMasters et al describes two reports affected, respectively, by mixed large and small vessel myelopathy and small vessel myelopathy, presenting multimetameric continuous lesions mainly involving grey matter. Both necrosis and inflammation have been observed. Until now VZV myelitis has been described as monofocal lesions, succeeding a zoster at the corresponding dermatome (12). In this severe, lifethreatening clinical setting, we would like to highlight the importance of taking into account also uncommon neurological complications of VZV reactivation, regardless of the apparent immune state of the patient, as early treatment could significantly improve the prognosis.

\section{References}

1. Gilden DH, Kleinschmidt-DeMasters BK, LaGuardia JJ, et al. Neurologic complications of the reactivation of Varicella-Zoster virus. N Engl J Med 2000;342(9):635-646. [PubMed: 10699164] 
2. Kleinschmidt-DeMasters BK, Amlie-Lefond C, Gilden DH. The patterns of VZV encephalitis. Hum Pathol 1996;27(9):927-938. [PubMed: 8816888]

3. Gray F, Belec L, Lescs MC, et al. Varicella-zoster virus infection of the central nervous system in the acquired immune deficiency sindrome. Brain 1994;117:987-999. [PubMed: 7953606]

4. Cinque P, Bossolasco S, Vago L, et al. Varicella-Zoster virus DNA in cerebrospinal fluid of patients infected with Human Immunodeficiency Virus: VZV disease of the Central Nervous System or subclinical reactivation of VZV infection? Clin Infect Dis 1997;25:634-639. [PubMed: 9314452]

5. Marchioni E, Ravaglia S, Piccolo G, et al. Postinfectious inflammatory disorders: subgroups based on prospective follow-up. Neurol 2005;65(7):1057-1065.

6. Aberle SW, Aberle JH, Steininger C, et al. Quantitative real time PCR detection of Varicella-zoster virus DNA in cerebrospinal fluid in patients with neurological disease. Med Microbiol Immunol 2005;194(12):7-12. [PubMed: 14997388]

7. Goldberg, JW. Primary angiitis of the central nervous system. In: Rolak, LA.; Harati, Y., editors. Neuroimmunology for the clinician. Butterworth Heienemann; 1997. p. 177-186.

8. Scheld, WM.; Whitley, RJ.; Marra, CM. Infections of the central nervous system. 3rd. Philadelphia: Lippincott Williams \& Wilkins; 2004.

9. Amlie-Lefond C, Kleinschmidt-DeMasters BK, Mahalingam R, et al. The vasculopathy of varicellazoster virus encephalopathy. Ann Neurol 1995;37:784-790. [PubMed: 7778852]

10. Sissoko D, Bellagra N, Dewilde A, et al. Varicella-zoster virus meningo-encephalomyelitis without skin eruption. Ann Biol Clin 1998;56:211-212.

11. Mancardi GL, Melioli G, Traverso F, et al. Zoster sine herpete causing encephalomyelitis. Italian J Neurol Sci 1987;8:67-70.

12. Devinsky O, Cho ES, Petito CK, et al. Herpes Zoster myelitis. Brain 1991;114(3):1181-1196. [PubMed: 1648419]

13. Russman AN, Lederman RJ, Calabrese LH, et al. Multifocal varicella zoster virus vasculopathy without rash. Arch Neurol 2003;60(11):1607-9. [PubMed: 14623735] 

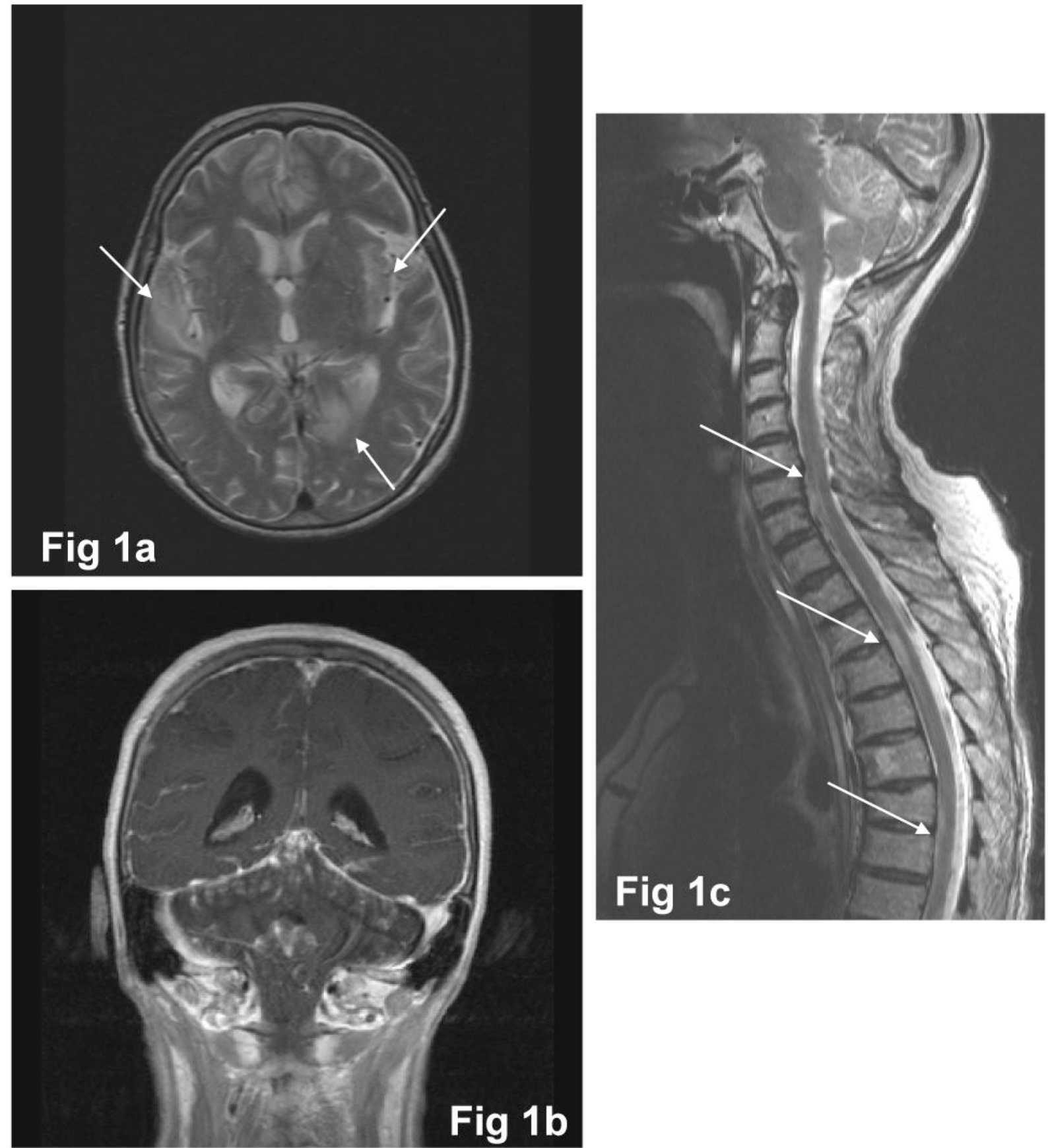

Fig 1.

Fig 1a: axial T2-weighted image: multiple bilateral hyperintense lesions (arrows), located at the right superior temporal gyrus, in the left paramedian parieto-occipital cortex and in the left external capsula

Fig 1b: coronal T1-weighted image post gadolinium injection: diffuse supra- and infratentorial contrast enhancement of meninges

Fig 1c: sagittal T2-weighted image: multiple cervico-dorsal hyperintense lesions (arrows), principally located in the anterior portion of the spine 


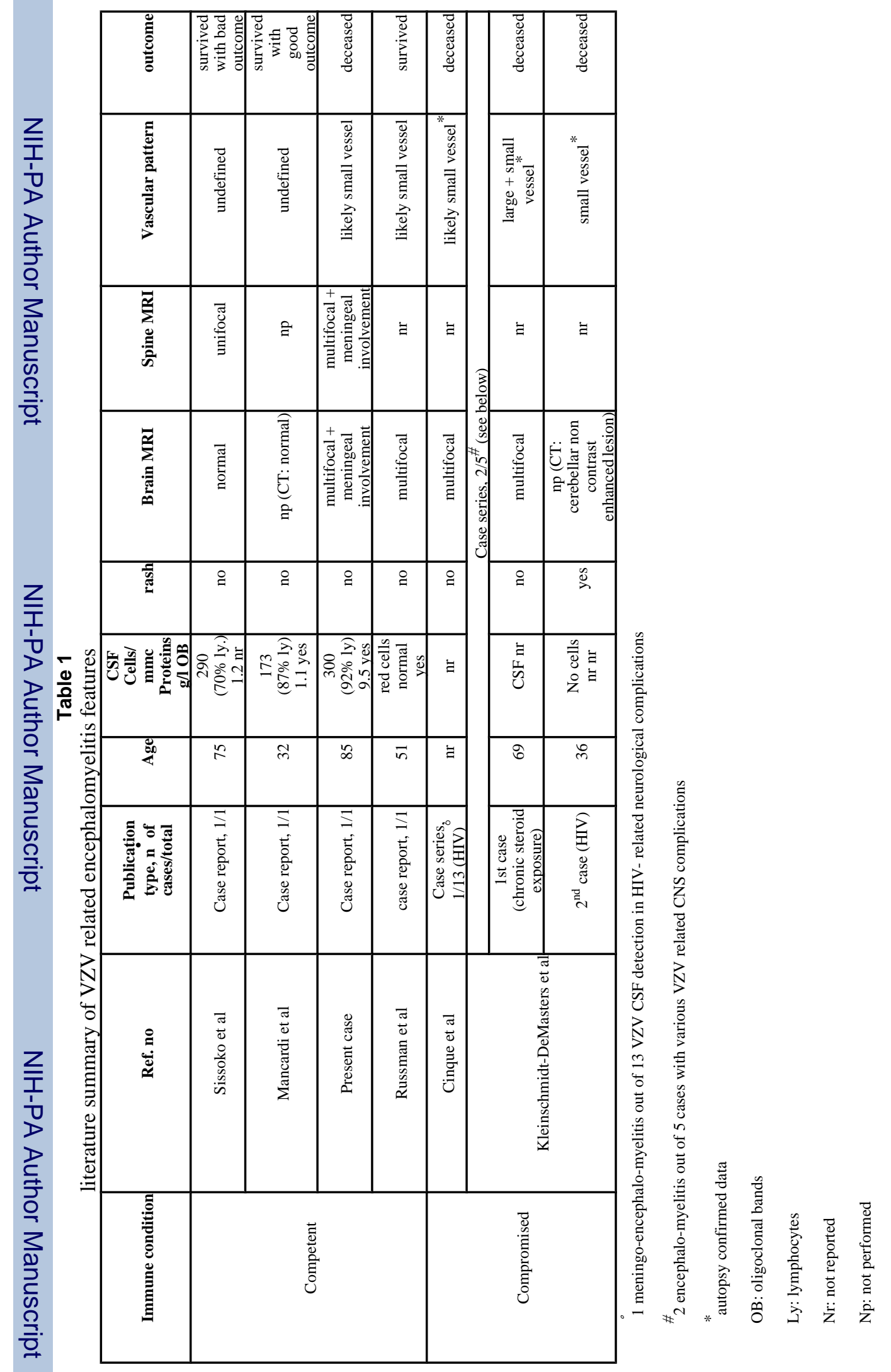

Neurol Sci. Author manuscript; available in PMC 2008 October 20. 